\title{
PROYECCIONES LABORALES DE LOS ESTUDIANTES DE ADMINISTRACIÓN DE EMPRESAS
} EMPLOYMENT PROJECTIONS FOR BUSINESS STUDENTS

\author{
Luz Dary Suarez Carvajalino ${ }^{*}$ \\ Wilder Quintero Quintero ${ }^{2}$
}

\author{
${ }^{1}$ Universidad Francisco de Paula Santander Ocaña, Colombia \\ https://orcid.org/0000-0003-2216-9926, E-mail: ldsuarezc@ufpso.edu.co \\ ${ }^{2}$ Grupo GIDSE, Universidad Francisco de Paula Santander Ocaña, Colombia \\ https://orcid.org/0000-0003-3260-6923 , E-mail: quinterowilder@ufpso.edu.co
}

Resumen: Resumen: La prospectiva laboral debe generarse desde los escenarios académicos de la Universidad. Con lo anterior, el presente artículo tiene como propósito conocer las proyecciones laborales de los estudiantes del noveno semestre de Administración de Empresas en la UFPSO. La metodología utilizada en la investigación fue cuantitativa de alcance descriptiva, aplicando la encuesta bajo el instrumento del cuestionario. Se concluye desde los resultados que más del $40 \%$ de los estudiantes esperan ingresar al sector comercial, asimismo, conseguir trabajo en menos o igual a un año después de graduado y, por último, el estudiante tiene la visión de aprender a dirigir y liderar procesos, desarrollar sus capacidades y talentos para poder obtener un buen salario y un reconocimiento por su labor ejecutada.

Palabras claves: Estudiante, Desempeño laboral, Proyección laboral.

Abstract: Employment prospects must be generated from the academic scenarios of the University. The purpose of the present article is to know the employment projections of the students of the ninth semester of Business Administration at UFPSO. The methodology of the investigation was quantitative with a descriptive scope by applyingthe survey under the questionnaire instrument. From the results, it is concluded that more than $40 \%$ of the students expect to enter the commercial sector, also, to get a job in less or equal to one year after graduation and, finally, the student has the vision to learn how to direct and lead processes, develop their skills and talents in order to obtain a good salary and recognition for their work.

Keywords: Student, Career projection, Job performance. 


\section{Introducción}

Los jóvenes activos en la Universidad, tienen muchos sueños y metas que esperan hacer realidad al culminar su etapa académica, sin embargo, en ellos existe la preocupación de conseguir u obtener el bienestar laboral y así mejorar la calidad de vida, desde luego, para lograr lo anterior, es necesario tener un trabajo formal. "Para la juventud actual, el trabajo y la profesión constituyen instrumentos en la búsqueda de la realización y la superación personal" (Narváez y Jiménez, 2017, p.13). De acuerdo a lo anterior, los universitarios esperan que las proyecciones laborales establecidas después de terminar la carrera de administración de empresas, permita el crecimiento profesional y ser referente o ejemplo de vida para las generaciones.

En el contexto empresarial, las ofertas o demandas laborales del siglo son inciertas e inestables, generando un rompimiento con la forma en la que habitualmente los estudiantes se vinculaban al mercado laboral, donde los jóvenes con formación universitaria se incorporaban de manera rápida y sin ningún esfuerzo al mercado de trabajo, en la actualidad se ven inmersos en complejas y múltiples formas de transición a la vida activa (Gonzales y Martínez, 2016). En la actualidad, al terminar una carrera universitaria no se asegura un cargo o trabajo, si no que se debe seguir estudiando más, y estar en una constante preparación.

Los estudiantes al tener unos objetivos definidos y una visión clara sobre la dinámica laboral a la que desean incorporarse, tendrá una mayor satisfacción ya que estará cumpliendo un rol donde se encuentra a gusto atendiendo sus obligaciones. Desde luego y desde la experiencia académica universitaria, surgen cuestiones sobre la percepción que cada uno de los estudiantes tiene sobre el rol que desempeñará en su futuro profesional y laboral; pero, sobre todo, cómo este afecta en los ámbitos y en su satisfacción personal y su eficiencia laboral (Biuza y Caicedo, 2012, p14). Con lo descrito, no existe un estudio sobre el plan laboral de los estudiantes futuros a terminar su carrera de administrador de empresas; lo que impide saber qué quieren hacer o cuál es el cargo de mayor interés para ejercer en una empresa. Incluso, Naranjo, 2009 (citado por Narváez y Jiménez, 2017) afirma:

Los estudiantes, y en general las personas, se motivan a realizar cosas (en nuestro caso, estudiar Administración de Empresas) y esforzarse por lograr un alto desempeño para alcanzar una meta si creen en su valor, si están seguras de que lo que harán contribuirá a lograrla y si saben que una vez que alcancen la meta recibirán una recompensa -es decir, un buen puesto en una buena empresa, un buen salario, prestigio-, de tal manera que el esfuerzo realizado ha valido la pena. (p.17)

Desde otra perspectiva, en la edad adulta de un ser humano la mayor parte del tiempo es dedicada a su trabajo, por esto siempre se quiere un ámbito laboral agradable, estable y empático, por ello, es importante visionar retos para tener claro los propósitos de vida profesional. Entonces, el fin del estudio es reconocer los factores o variables de interés por los estudiantes en materia laboral y las posibles situaciones para ingresar una vez culmine los estudios académicos, debe ser prioritario para avanzar en el desarrollo, mejora y funcionalidad de la educación superior (Gonzales y Martínez, 2016).

Finalmente, con la investigación se presentan las proyecciones laborales de los estudiantes del noveno semestre de administración de empresas de la Universidad 
Francisco de Paula Santander Ocaña, generando información valiosa y útil, paraque las autoridades competentes de la Educación superior puedan diseñar estrategias que permitan a los administradores tener objetivos claros, unavisión a seguir, y una mayor satisfacción el logro de sus metas.

\section{Marco Teórico}

Para el desarrollo de la investigación se tuvo en cuenta las teorías de las relaciones humanas, teoría de la productividad laboral, la teoría de Herzberg y la pirámide Maslow (Romaní y Sáenz, 2014, p.15).

Las relaciones humanas es una teoria, donde se presume que las personas fueran valoradas en el trabajo y permitiera al empleado tener autonomia, tomar decisiones y lograr más confianza hacia a la personas, que elaboran y aportan grandes cosas a la entidad.

Teoría de Herzberg induce sobre la motivación desde los diversos factores causantes de satisfacción e insatisfacción en el trabajo. Donde identificó dos factores que influyen en la motivación en el trabajo. A éstos les llamó factores: "ambientales" y "motivadores". El primero se puede considerar como el ámbito o contexto del trabajo (antecedente), y el segundo el contenido del trabajo (consecuente) (Domínguez, 2008). Desde luego, al tener los factores de motivación y los factores ambientales, el trabajador puede ejercer su deber con mayor satisfacción. La teoría de Herzberg, nos dice que las necesidades de bajo nivel deben de estar cubiertas para que no exista insatisfacción.
Por otro lado, es importante para tener un autoconocimiento, lo cual, nos permite estar en una mejora continua para realizar la actividades propuestas o establecidas. Según Marchant y Quijano (2006) citado por Marvel, Mirza; Rodríguez, Carlos; Núñez, Miguel (2011) considera que la productividad del factor humano es un factor de éxito para el logro de los objetivos corporativos, de manera que logran el desempeño económico la permanencia, siendo una cualidad clave para la selección de personas jóvenes en el mundo laboral.

Finalmente, desde la pirámide Maslow, se reconocen cinco niveles, en los cuales se encuentran las necesidades básicas del individuo de manera jerárquica, sin embargo, el de mayor importancia para el estudio es la autorrealización, es decir, motivación de crecimiento, donde se encuentra sentido a la vida por medio del desarrollo potencial de una actividad (Marroquín, 2011).

\section{Metodología}

La investigación de tipo cuantitativo tiene un alcance descriptivo, puesto que en dichas investigaciones se recolectarán datos. Arias (2012) define la investigación como la caracterización de un hecho, fenómeno, individuo o grupo, con el fin de establecer su estructura o comportamiento.

La encuesta se realizó a 21 estudiantes inscritos en la Asignatura Juegos Gerenciales perteneciente al noveno semestre según la malla curricular del programa de Administración de la universidad Francisco de Paula Santander Ocaña y que tienen la iniciativa de esperar ser contratado y no emprendedor, finalmente, para recolectar la información se trabajó el cuestionario y la lista de asistencia para el seguimiento. 


\section{Resultados}

Se presentan los siguientes resultados del estudio mediante tablas para facilitar la presentación de los datos suministrados por la muestra objetiva.

Tabla 1. Empresas de interés para los futuros administradores

\begin{tabular}{lccc}
\hline & Frecuencia & Porcentaje \\
\cline { 1 - 1 } Empresas & 10 & $47 \%$ \\
comerciales & 10 & $29 \%$ \\
Empresas industriales & 6 & $24 \%$ \\
Empresas de servicios & 5 & $100 \%$ \\
Total & 21 & \\
\hline
\end{tabular}

Fuente: Elaboración propia.

Con los datos se observa que más del $40 \%$ desean vincularse al sector comercial a través de la venta de productos por diferentes redes y canales, el $29 \%$ trabajar en el sector industrial, especialmente, multinacionales del país. Finalmente, el $25 \%$ desarrollar ejercicios en el sector de servicios.

Tabla 2. Situación de los estudiantes para ingresar al ejercicio laboral

\begin{tabular}{lcc}
\hline & Frecuencias & Porcentaje \\
\hline $\begin{array}{l}\text { Tiene confianza, } \\
\text { conocimiento y } \\
\text { excelentes habilidades } \\
\text { para cargos laborales. }\end{array}$ & 9 & $43 \%$ \\
$\begin{array}{l}\text { Está preocupado(a) por } \\
\text { su futuro laboral debido } \\
\text { a aumento de } \\
\text { desempleo, falta de } \\
\text { oportunidades, } \\
\text { desconocimiento, poca } \\
\text { práctica, entre otros. }\end{array}$ & 10 & $48 \%$ \\
NS/NR & 2 & $6 \%$ \\
Total & $\mathbf{2 1}$ & $\mathbf{1 0 0 \%}$ \\
\hline
\end{tabular}

Fuente: Elaboración propia.
Siguiendo los datos se conoce que un $48 \%$ de los estudiantes están preocupados por su futuro debido al aumento de desempleo, falta de oportunidades, desconocimiento y poca práctica lo cual es un reto para los estudiantes en un tiempo ya graduados, por otro lado, con un $43 \%$ los estudiantes tienen confianza, conocimiento y habilidades para cargos laborales y $\tan$ solo con $6 \%$ no se preocupa hasta el momento por el porvenir profesional.

Tabla 3. Tiempo de búsqueda laboral

\begin{tabular}{lcc} 
& Frecuencia & Porcentaje \\
\cline { 1 - 2 } De 1 año o menos & 15 & \\
De 1 año a 2 años & 6 & $29 \%$ \\
Total & 21 & $100 \%$ \\
\hline
\end{tabular}

Fuente: Elaboración propia

Se observa en los datos que el $71 \%$ de los estudiantes cree que en un tiempo de 1 año o menos podrán encontrar un trabajo en la respectiva área, el 29\% manifestó entre 1 a 2 años debido a que las tasas de desempleo y de egresados aumentan con el transcurrir de los trimestres

\section{Conclusiones}

Con este estudio se conocieron las proyecciones laborales de los estudiantes del noveno semestre del programa de Administración de Empresas de la UFPSO, y se generó información valiosa y útil para la universidad, donde el $48 \%$ de los estudiantes de Administración de Empresas se encuentran preocupados para ejercer excelentemente las labores profesionales en el sector empresarial y con la comunidad. Sin embargo, es claro que, ante la vinculación a nuevos procesos, ciclos, escenarios y aprendizajes de tipo ocupacional, surjan interrogantes, pensamientos e incertidumbre para afrontar las situaciones mencionadas. 
Por otra parte, los estudiantes esperan conseguir trabajo en menos o igual a un año después de graduado o titulado, por tanto, existe el deseo de edificar su visión, pero a su vez asegurar un futuro laboral, asimismo, aprender cosas nuevas, dirigir y liderar procesos trabajar en actividades de su gusto, desarrollar sus capacidades y talentos que poder obtener un buen salario $y$ reconocimiento de su ejercicio.

Finalmente, los futuros administradores manifiestan promover la experticia desde la academia para fortalecer más las competencias blandas, de hecho, la población estudiantil espera desarrollar lascompetencias avanzadas través del semestre de prácticas empresariales, a su vez, entender el comportamiento interno y externo del mercado profesional.

\section{Referencias}

Arias, F. (2012). Introducción a la metodología científica . EPISTEME, C.A.

Biuza, B., \& Caicedo, W. (2012). EXPECTATIVAS LABORALES Y EMPRESARIALES DE LOS

ESTUDIANTES. Obtenido de http://bibliotecadigital.univalle.edu.c o/bitstream/10893/10709/1/CB0552863.pdf

Marvel Cequea, Mirza, \& Rodríguez Monroy, Carlos, \& Núñez Bottini, Miguel Angel (2011). La productividad desde una perspectiva humana: Dimensiones y factores. Intangible Capital, 7(2),549- 584.[fecha de Consulta 27 de Mayo de2020]. ISSN: 2014-3214. Disponible

en: https://www.redalyc.org/articulo.oa?i $\mathrm{d}=549 / 54921605013$

Moscoso, Y. (2017). Obtenido de https://lasillavacia.com/sillallena/red-de-la-educacion/historia/laimportancia-del-reconocimiento$\underline{61816}$

Romaní, L., \& Sáenz, C. (2014). Obtenido de https://www.academia.edu/8569162/ Enfoque humanistico_de la_Admini stracion

Domínguez, P. (2008). Obtenido de http://www.eumed.net/librosgratis/2006a/prd/6c.htm

Marroquín, N. (2011). Obtenido de http://biblioteca.usac.edu.gt/tesis/03/ 03_3861.pdf

Azcoiti, R. (2008). Obtenido de http://www.memoria.fahce.unlp.edu. ar/tesis/te.594/te.594.pdf 